Article

\title{
Biohybrid Nanostructured Iron Oxide Nanoparticles and Satureja hortensis to Prevent Fungal Biofilm Development
}

\author{
Ion Anghel ${ }^{1,2}$, Alexandru Mihai Grumezescu ${ }^{3}$, Alina Maria Holban ${ }^{4, *}$, Anton Ficai ${ }^{3}$, \\ Alina Georgiana Anghel ${ }^{1,2}$ and Mariana Carmen Chifiriuc ${ }^{4}$
}

1 Otorhinolaryngology, Carol Davila University of Medicine and Pharmacy, Traian Vuia no 6, Bucharest 020956, Romania; E-Mails: ionangheldoc@yahoo.com (I.A.); dr_alina.anghel@yahoo.com (A.G.A.)

2 R \& D Department, Doctor Anghel Medical Center, Theodor Sperantia Street, Bucharest 30932, Romania

3 Department of Science and Engineering of Oxide Materials and Nanomaterials, Faculty of Applied Chemistry and Materials Science, Politehnica University of Bucharest, Bucharest 011061, Romania; E-Mails: grumezescu@yahoo.com (A.M.G.); anton_ficai81@yahoo.com (A.F.)

4 Department of Microbiology and Immunology, Faculty of Biology, University of Bucharest, Bucharest 060101, Romania; E-Mail: carmen_balotescu@yahoo.com

* Author to whom correspondence should be addressed; E-Mail: alina_m_h@yahoo.com; Tel./Fax: +40-21318-1577.

Received: 17 June 2013; in revised form: 5 August 2013 / Accepted: 23 August 2013 / Published: 4 September 2013

\begin{abstract}
Cutaneous wounds are often superinfected during the healing process and this leads to prolonged convalescence and discomfort. Usage of suitable wound dressings is very important for an appropriate wound care leading to a correct healing. The aim of this study was to demonstrate the influence of a nano-coated wound dressing (WD) on Candida albicans colonization rate and biofilm formation. The modified WD was achieved by submerging the dressing pieces into a nanofluid composed of functionalized magnetite nanoparticles and Satureja hortensis (SO) essential oil (EO). Chemical composition of the EO was established by GC-MS. The fabricated nanostructure was characterized by X-ray Diffraction (XRD), Transmission Electron Microscopy (TEM), Differential Thermal Analysis (DTA) and Fourier Transform-Infrared Spectroscopy (FT-IR). The analysis of the colonized surfaces using (Scanning Electron Microscopy) SEM revealed that C. albicans adherence and subsequent biofilm development are strongly inhibited on the
\end{abstract}


surface of wound dressing fibers coated with the obtained nanofluid, comparing with regular uncoated materials. The results were also confirmed by the assay of the viable fungal cells embedded in the biofilm. Our data demonstrate that the obtained phytonanocoating improve the resistance of wound dressing surface to $C$. albicans colonization, which is often an etiological cause of local infections, impairing the appropriate wound healing.

Keywords: nano-modified wound dressing; Satureja hortensis essential oil; fungal biofilm; Candida albicans; magnetite nanoparticles; iron oxide

\section{Introduction}

The use of modified nanostructured surfaces for the design of film-coated surfaces of solid and fiber-based materials provide a new approach to prevent or disrupt the formation of microbial biofilms [1]. Candida albicans associated wound infections are frequently associated with burns $(28 \%)$ [2], and less frequently with non-surgical epithelial injuries (0.8\%) [3]. A significant increase was seen in infections attributable to $C$. albicans in surgical site postoperative infections [4]. One of the major complications in C. albicans wound infections is biofilm formation, since microorganisms embedded in biofilms are hundred times more resistant to antifungal compounds $[5,6]$, the infection being therefore difficult to eradicate $[7,8]$.

Iron oxide based nanosized materials are of great interest for the biomedical field due to their excellent properties [9], derived from their intrinsic magnetic nature, as well as from the enhanced physico-chemical properties, such as ultra small and controllable size, large surface area to mass ratio, high reactivity, and functionalizable structure [10]. Magnetite $\left(\mathrm{Fe}_{3} \mathrm{O}_{4}\right)$ has been widely studied for biomedical applications in biological separations [11], drug delivery and targeting [12-15], magnetic resonance imaging [16], hyperthermia [17], cancer treatment [18,19], stabilization of essential oils [20], inhibition of microbial colonization [21] and ferrofluids [9,22]. The magnetic nanoparticles as delivery nanosystems are considered effective new tools to tackle the current challenges in treating infectious diseases, by improving the therapeutic index of antimicrobial drugs, and diminishing the local and systemic side effects including cutaneous irritation, peeling, scaling and gut flora reduction [23].

The essential oils are an interesting alternative for the antimicrobial therapy, acting by multiple mechanisms, including cell wall damages, inhibiting the cell wall or protein synthesis, or interfering with intermediary metabolisms or DNA/RNA synthesis/function [24,25]. However, the therapeutic effects of the essential oils can be impaired by their high volatility, highlighting the necessity of vectoring stabilizing systems. Satureja hortensis $(\mathrm{SH})$ is an annual, herbaceous plant belonging to the family Labiatae [26]. The main components of the essential oils of this plant are the carvacrol, thymol, p-cymene, $\beta$-caryophyllene, linalool and other terpenoids [27]. The essential oils isolated from various species of Satureja have biological properties such as antimicrobial, antiviral, antispasmodic and antidiarrhoeal [28]. 
Routinely used wound dressings are essential in any wound care. An ideal wound dressing should be completely biocompatible and skin-friendly, but also unpermissive for microbial development on the wound lesion. We have previously reported the obtaining of modified textile wound dressings coated with functionalized magnetite nanoparticles, with improved antimicrobial and antibiofilm properties, towards both bacterial and fungal strains. Also the functionalized magnetite nanoparticles proved to act as an efficient delivery system for essential oils and some of their major components.

In this study we report the fabrication, characterization and bioevaluation of a novel wound dressing coating, containing iron oxide nanoparticles and Satureja hortensis essential oil. These modified wound dressings exhibited improved antimicrobial properties, preventing fungal colonization and biofilm development.

\section{Results and Discussion}

Among the promising approaches to combat biofilm infections is the generation of surface modification of devices to reduce microbial attachment and biofilm development, as well as incorporation of antimicrobial agents to prevent colonization. The essential oils represent a promising alternative to antimicrobial substances, due to their multiple advantages, such as: an easy way of obtaining low mammalian toxicity, quick biodegradability and low probability for the development of bacterial resistance [29]. Recent studies have shown that nanoparticles can be used for the stabilization and prolonged delivery of essential oils and for the enhancement of their activity at the site of infection, thus surpassing some of the main drawbacks for conventional antimicrobial agents, which are the development of multiple drug resistance and adverse side effects.

Our previous studies have demonstrated that Rosmarinus officinalis essential oil-coated magnetic nanoparticles strongly inhibited the adherence ability and biofilm development of $C$. albicans and C. tropicalis clinical strains [30] on the catheter surface, and usnic acid-coated magnetic nanoparticles strongly inhibited the adherence ability and biofilm development of Staphylococcus aureus on the coverslips surface, opening new perspectives for the design of antimicrobial and antibiofilm surfaces, based on hybrid functionalized nanostructured biomaterials [31]. In this paper we have investigated the antifungal biofilm properties of a modified wound dressing with hybrid nano-coating based on magnetic nanoparticles and SH essential oil.

The SH essential oil isolated by microwave assisted distillation from the aerial parts of $S$. hortensis, was found to be a yellow liquid and the main components were presented in Table 1.

Table 1. GC-MS analysis of Satureja hortensis (SH) essential oil.

\begin{tabular}{cccc}
\hline No. & Compound & Retention Index $^{\mathbf{1}}$ & $\mathbf{( \% )}$ \\
\hline 1 & $\alpha$-thujene & 927 & 1 \\
2 & $\alpha$-pinene & 940 & 0.9 \\
3 & $\beta$-pinene & 984 & 0.7 \\
4 & myrcene & 1000 & 1.3 \\
5 & $\alpha$-terpinene & 1019 & 3.6 \\
6 & p-cymene & 1028 & 4.8 \\
7 & $\gamma$-terpinene & 1057 & 38.7 \\
8 & linalool & 1103 & 0.9 \\
\hline
\end{tabular}


Table 1. Cont.

\begin{tabular}{cccc}
\hline No. & Compound & Retention Index 1 & $\mathbf{( \% )}$ \\
\hline 9 & carvacrol & 1301 & 46.9 \\
10 & $\beta$-caryophyllene & 1413 & 0.1 \\
11 & $\beta$-bisabolone & 1504 & 0.7 \\
\hline \multicolumn{2}{c}{${ }^{1}$ RI is the retention indices in elution order from DB-5 column. }
\end{tabular}

Eleven components were identified in the essential oil of SH. The main constituents of the essential oil of SH are the carvacrol (46.9\%), $\gamma$-terpinene (38.7\%), p-cymene (4.8\%), $\alpha$-terpinene $(3.6 \%)$ and myrcene $(1.3 \%)$. Variation in essential oil content and composition of SH essential oils from different origins has been reported in the literature [32]. The reported results support previous literature data [33].

XRD pattern (Figure 1) show that the MNP@18 are well-crystalline and exhibit diffraction peaks corresponding to (111), (220), (311), (400), (511) and (440) planes of cubic crystal system. The position and relative intensity of diffraction peaks are same with the standard data for bulk magnetite (JCPDS file No. 19-0629) which further indicates the purity of synthesized MNP@18. XRD supports the data previously reported [34].

Figure 1. XRD pattern of MNP@18.

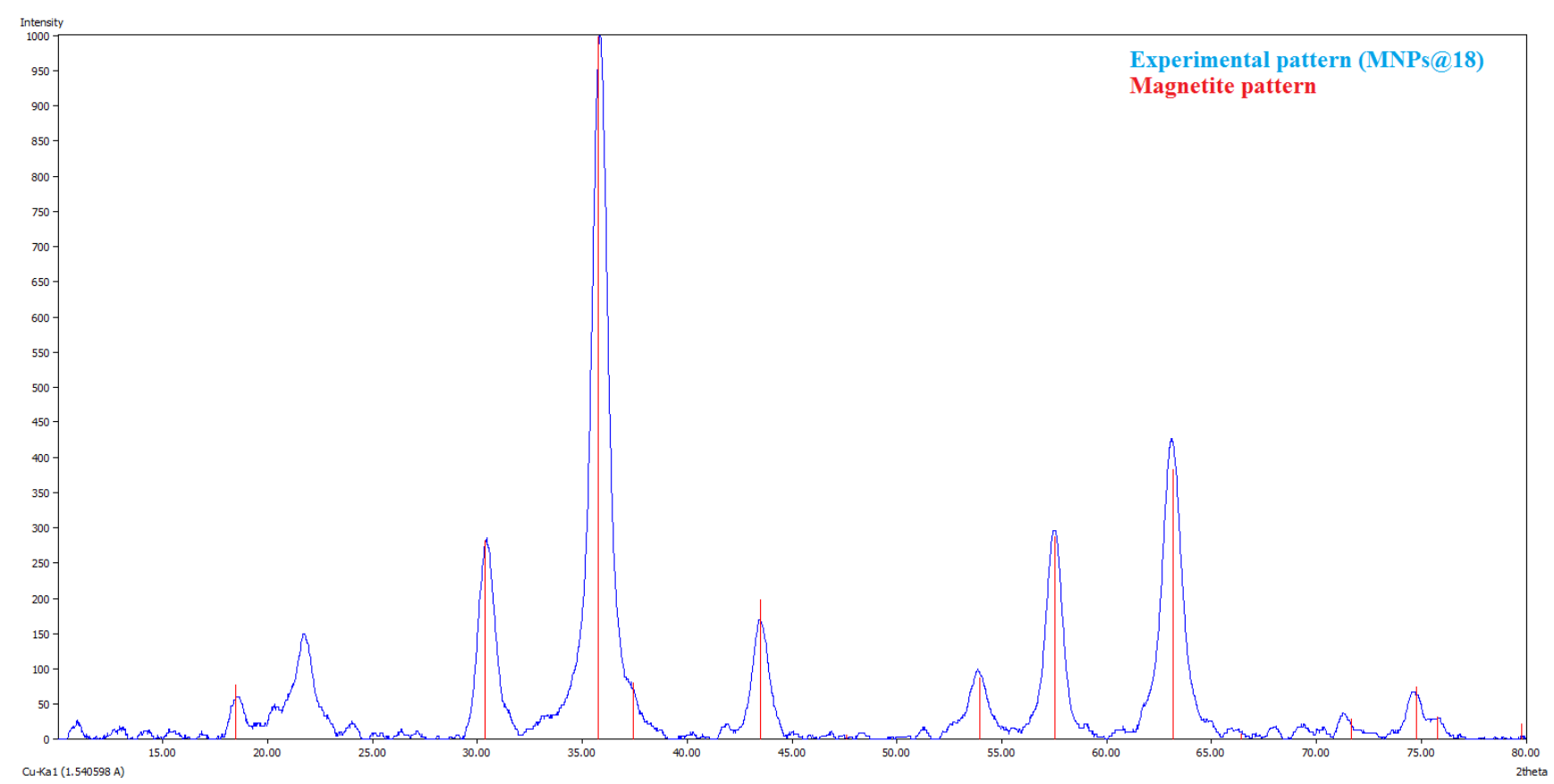

The TEM images of the MNP@18 were used to determine the shape, size and uniformity of the particles. Figure 2a,b that the particles are spherical and polydispersed with an average size of $10 \mathrm{~nm}$.

The TGA thermograms revealed continuous weight loss for $\mathrm{C} 18$ and $\mathrm{SH}$ (Figure 3). The weight losses are 23.45\% for MNP@18, and 37.82\% for of MNP@18-SH. The results confirmed the attachment and stabilization of the volatility of the SH essential oil on MNP@18 surface. The SH essential oil content was estimated as the difference between the weight loss for the region at approximately $500{ }^{\circ} \mathrm{C}$ for MNP@18-SH and MNP@18, and it was approximately $14.37 \%$. 
Figure 2. Transmission electron microscopy (TEM) images of MNPs@18 (a) and histogram showing the size distribution of the MNPs@18 (b).
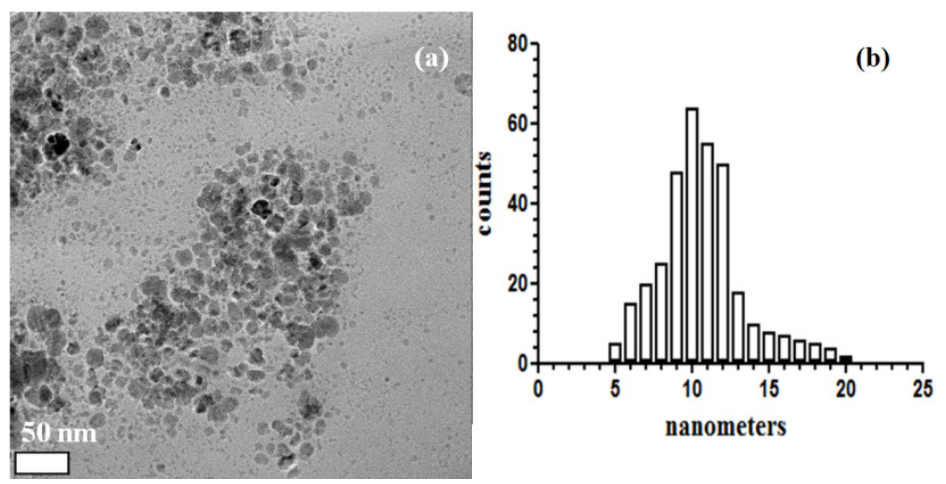

Figure 3. Thermogravimetric (TG) analysis of MNP@18-SH and MNP@18.

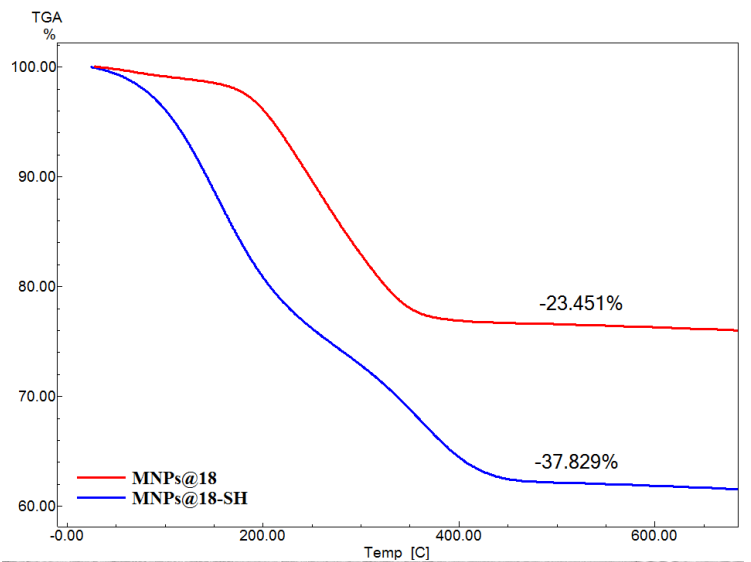

The FT-IR spectrum of modified wound dressing (Figure 4) showed characteristic band of iron oxide at $\sim 545 \mathrm{~cm}^{-1}$ attributed to the stretching vibration of $\mathrm{Fe}-\mathrm{O}$ bonds [35] and characteristic bands assigned to stretching vibration of $\mathrm{C}-\mathrm{H}$ from organic coating $(\mathrm{C} 18)$ at about 2919 and $2851 \mathrm{~cm}^{-1}$.

Figure 4. Fourier Transform-Infrared Spectroscopy (FT-IR) spectra of (modified) wound dressing (WD).

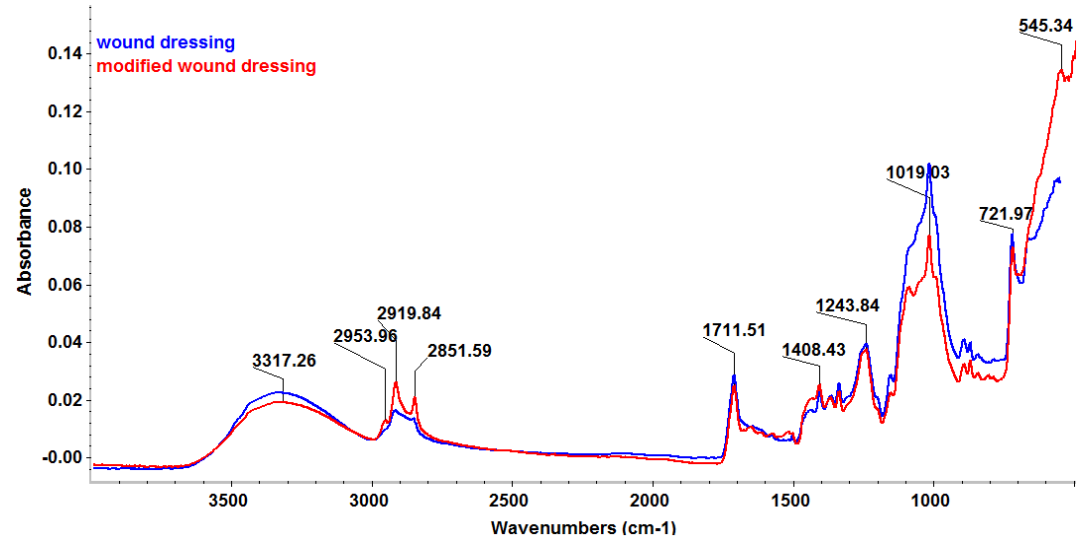

Recent studies have proved that the major compounds identified in the composition of SH essential oil exhibit antimicrobial properties [36,37]. The essential oils extracted from different aerial parts of 
SH (i.e., budding, full flowering, immature fruit, and ripened fruit stages) exhibited strong antibacterial activities against a wide range of bacterial and fungal strains (including S. aureus, E. coli and C. albicans), clearly demonstrating their potential to be used in the management of microbial infections [38-41]. Other studies have shown that the amount of EOs extracted from different air dried samples were quite similar varying from $1.8 \%$ in case of ripened fruit stage to $2.5 \%$ at full flowering, as well as the chemical composition, which was quite consistent, the $\delta$-terpinene being the major compound of the EO at all developmental stages, except the ripened fruit stage when it was replaced by carvacrol [37]. These two compounds have been also found in major percentages in the composition of the EO used in the present study.

The SH essential oils could also contribute to the development of environmentally safer alternatives to protect the spoilage of food products from pathogenic and saprophytic fungi, by inhibiting the mycelial growth of Alternaria mali Roberts and Botrytis cinerea Pers fungi, and also exhibiting a fungicidal effect against these phytopathogenic species [42].

In our study, viable cell counts results revealed that MNP@18-SH coated WD exhibited significant antimicrobial properties, disrupting fungal adherence and biofilm formation.

Figure 5. Graphic representation of viable cell counts analysis after removing C. albicans biofilm embedded cells at $24 \mathrm{~h}, 48 \mathrm{~h}$ and $72 \mathrm{~h}$ post inoculation of control and nanobiocoated WDs. $* p<0.05, * * p<0.01, * * * p<0.001$ samples $v s$. WD control.

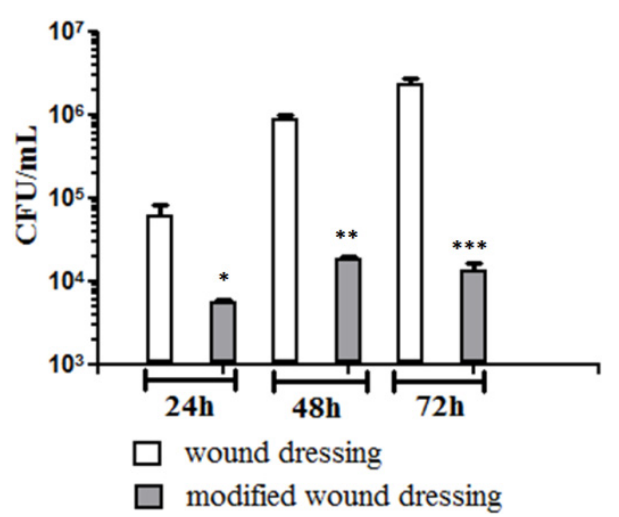

On the regular WD fibers, the kinetics of $C$. albicans biofilm registered an ascending trend from $24 \mathrm{~h}$ to $72 \mathrm{~h}$, as revealed by the increasing number of biofilm embedded viable cells. The biofilm formation on the nanomodified WDs was impaired in its early as well as mature phases, quantified at $24 \mathrm{~h}, 48 \mathrm{~h}$ and $72 \mathrm{~h}$ (Figure 5), therefore the nano-coating stabilizes and preserves the antimicrobial activity of the essential oil. Viable cell counts data were also confirmed by the microscopic examination of the biofilm architecture and development. The scanning electron microscopy images showed that $C$. albicans formed yeast microcolonies embedded in an extracellular mathrix on the surface of control WDs, this ability being abolished when using nanobiocoated WD surfaces colonized for $24 \mathrm{~h}, 48 \mathrm{~h}$ and $72 \mathrm{~h}$ (Figure 6). The antimicrobial activities of SH essential oil could be explained by the high content in phenolic compounds, such as thymol and carvacrol or p-cymene, whose antimicrobial effect is due to damages induced in the membrane integrity, causing changes in $\mathrm{pH}$ homeostasis and also in the equilibrium of inorganic ions [39]. Although p-cymene is not reported in the literature as having antimicrobial activity, it increases the antimicrobial activity of thymol or 
carvacrol [43], by destabilizing the cytoplasmic membrane of microbial cell [44]. This synergic activity of the active compounds has motivated us to use the essential oil whole extract instead of individual compounds. The antibiofilm activity of the modified wound dressing was preserved on the entire duration of the experiment, proving that the nanoparticles acted as an efficient stabilization and long lasting release vehicle for the essential oil.

Figure 6. SEM micrographs indicating the $C$. albicans biofilm development comparatively on control WDs (after $24 \mathrm{~h}-\mathbf{a}_{1}, 48 \mathrm{~h}-\mathbf{b}_{1}$ and $72 \mathrm{~h}-\mathbf{c}_{1}$ incubation time) and on MNP@18-SH coated WDs (after $24 \mathrm{~h}-\mathbf{a}_{2}, 48 \mathrm{~h}-\mathbf{b}_{2}$. and $72 \mathrm{~h}-\mathbf{c}_{2}$ incubation) (2500×). The Candida biofilms developed on the coated WDs are strongly damaged and drastically reduced.

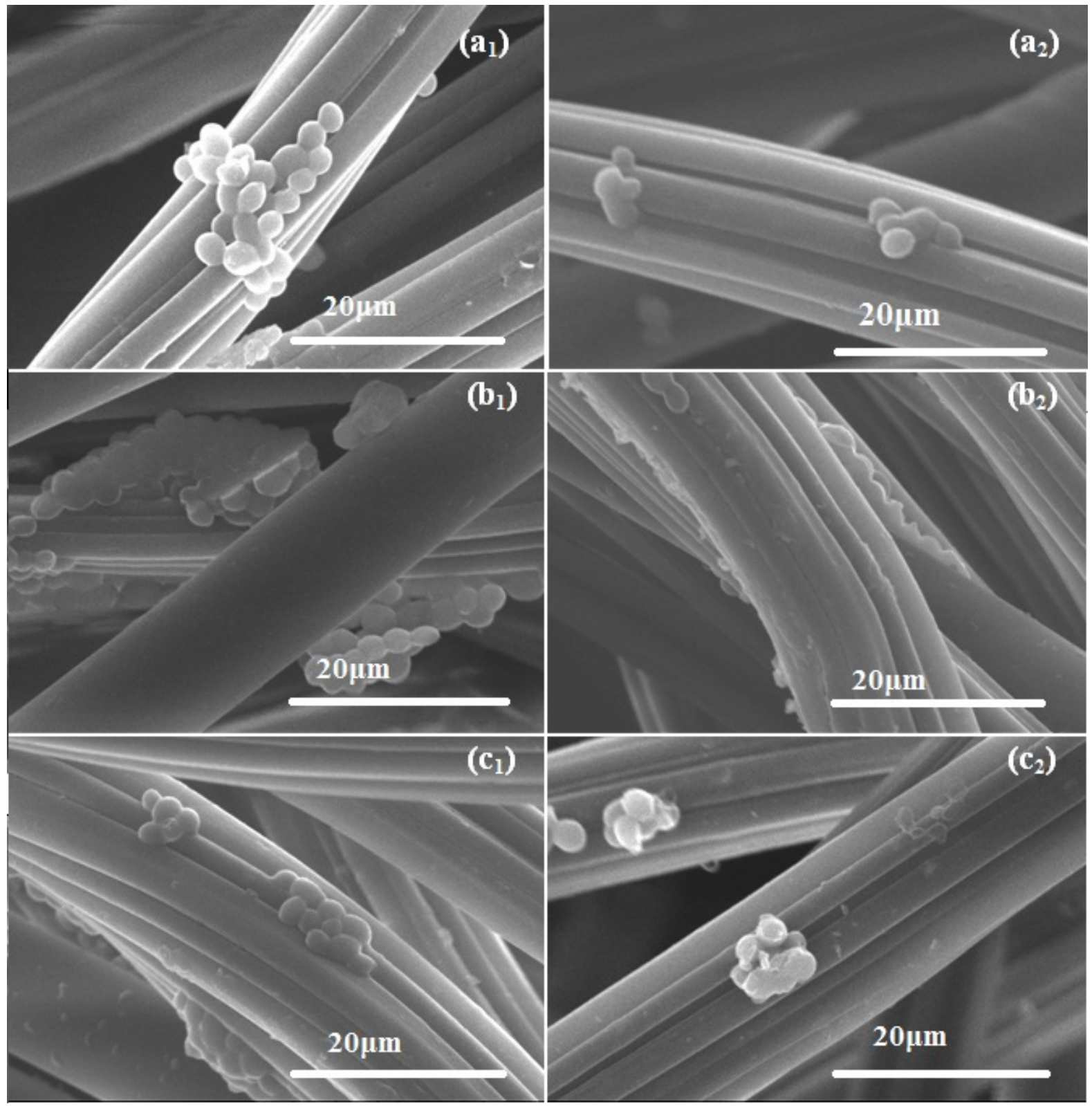




\section{Experimental Section}

\subsection{Materials}

All chemicals were used as received. $\mathrm{FeCl}_{3}, \mathrm{FeSO}_{4} \cdot 7 \mathrm{H}_{2} \mathrm{O}, \mathrm{NH}_{4} \mathrm{OH}(25 \%)$, and $\mathrm{CH}_{3} \mathrm{OH}$ were purchased from Sigma-Aldrich ChemieGmbh (Munich, Germany). General-use $10 \times 10 \mathrm{~mm}$ rayon/polyester based wound dressings were provided from Doctor Anghel's Medical Center.

\subsection{Synthesis of Functionalized Magnetite Nanostructure}

Magnetite nanostructure was prepared by wet chemical precipitation from aqueous iron salt solutions by means of alkaline media $[13,45,46]$. Synthesis of functionalized magnetite nanostructure involves several steps. Briefly, magnetic nanoparticles of approximately $10 \mathrm{~nm}$ diameter were precipitated in alkaline solution of sodium stearate $\left(\mathrm{C}_{18}\right)$ from solution of $\mathrm{Fe}(\mathrm{II})$ and $\mathrm{Fe}(\mathrm{III})$ according to our recently published paper [47]. After the precipitation of functionalized magnetite nanocrystals (MNP@18), it was repeatedly washed with methanol and separated with a strong $\mathrm{NdFeB}$ permanent magnet.

\subsection{Extraction and Analysis of Satureja hortensis (SH) Essential Oil}

The essential oil microwave assisted extraction was performed in a Neo-Clevenger type apparatus and its chemical composition was settled by GC-MS analysis. Gas chromatographic analysis was performed using an Agilent 6890 Series GC System (Agilent Technologies Inc., Santa Clara, CA, USA) gas chromatograph fitted with a splitless injector for a low background under a column head pressure of $12.5 \mathrm{psi}$ and $\mathrm{H}_{2}$ as carrier gas at a flow rate of $1.2 \mathrm{~mL} / \mathrm{min}$. Oven temperature was programmed from $50{ }^{\circ} \mathrm{C}$ to $300{ }^{\circ} \mathrm{C}$ at $5{ }^{\circ} \mathrm{C} / \mathrm{min}$. Injector and detector temperatures were $250{ }^{\circ} \mathrm{C}$. A capillary column DB5-MS fused-silica J\&W Scientific Inc. (Krackeler Scientific, Inc., Albany, NY, USA) was used (30 $\mathrm{m} \times 0.25 \mathrm{~mm}$ i.d.; $0.25 \mu \mathrm{m}$ film). Detection was carried out with a 5973 mass-selective single quadrupole detector (Agilent technologies Inc., Santa Clara, CA, USA). Operation control and the data process were carried out by Agilent Technologies ChemStation software (Santa Clara, CA, USA). The mass spectrometer was calibrated before use with perfluorotributylamine (PFTBA) as a calibration standard.

\subsection{Fabrication of Functionalized Magnetite Biohybrid Nanostructure}

MNP@18 (100 mg) and $100 \mu \mathrm{L}$ of SH were solubilized in $2 \mathrm{~mL}$ of chloroform and mixed until complete evaporation of chloroform was reached. According to our previous published work [20], we observed that this ratio (100 mg of MNP@18 and $100 \mu \mathrm{L}$ of essential oil) is the most appropriate for the efficient stabilization of the most essential oils.

This step was repeated three times for the uniform loading of HS in the MNP@18. After 72 h the prepared MNP@18-SH was analyzed by TGA to estimate the amount of SH essential oil entrapped into the MNP@18 [30,31]. 


\subsection{Fabrication of Modified Wound Dressing}

After $72 \mathrm{~h}$ of drying at room temperature, the layer of MNP@18-SH on the wound dressing material was achieved by submerging the dressing pieces $(10 \times 10 \mathrm{~mm})$ in $5 \mathrm{~mL}$ of MNP@18-SH fluid (MNP@18-SH: $\mathrm{CHCl}_{3}=1 \mathrm{mg} / \mathrm{mL}$ ), and then the dressing pieces have been extemporaneously dried at room temperature. The rapid drying was facilitated by the convenient volatility of chloroform. The modified wound dressing specimens were sterilized by ultraviolet irradiation for $20 \mathrm{~min}$.

\subsection{Characterization}

\subsubsection{TEM}

The transmission electron microscopy (TEM) images were obtained on finely powdered samples using a Tecnai ${ }^{\mathrm{TM}}$ G2 F30 S-TWIN high resolution transmission electron microscope from FEI Company (Hillsboro, OR, USA). The microscope was operated in transmission mode at $300 \mathrm{kV}$ with TEM point resolution of $2 \AA$ and line resolution of $1 \AA$. The fine powder was dispersed into pure ethanol and ultrasonicated for $15 \mathrm{~min}$. After that, diluted sample was put onto a holey carbon-coated copper grid and left to dry before TEM analysis.

\subsubsection{XRD}

X-ray diffraction analysis was performed on a Shimadzu XRD 6000 diffractometer at room temperature. In all the cases, $\mathrm{Cu} \mathrm{K} \alpha$ radiation $(\lambda=15,406 \AA$ at $15 \mathrm{~mA}$ and $30 \mathrm{kV})$ was used. The samples were scanned in the Bragg angle $2 \theta$ range of $10-80$ degree.

\subsubsection{FT-IR}

A Nicolet 6700 FT-IR spectrometer (Thermo Nicolet, Madison, WI, USA) connected to the software of the OMNIC operating system (Version 8.2; Thermo Nicolet, Madison, WI, USA) was used to obtain FT-IR spectra of the modified wound dressings. The samples were placed in contact with attenuated total reflectance (ATR) on a multibounce plate of ZnSe crystal at controlled ambient temperature $\left(25{ }^{\circ} \mathrm{C}\right)$. FT-IR spectra were collected in the frequency range of $4000-650 \mathrm{~cm}^{-1}$ by co-adding 32 scans and at a resolution of $4 \mathrm{~cm}^{-1}$ with strong apodization. All spectra were ratioed against a background of an air spectrum.

\subsubsection{TG analysis}

The thermogravimetric (TG) analysis of the MNP@18 and MNP@18-SH was followed with a Netzsch TG 449C STA Jupiter instrument (Netzsch, Selb, Germany). Samples were screened with 200 mesh prior to analysis, placed in an alumina crucible, and heated at $10 \mathrm{~K} \mathrm{~min}{ }^{-1}$ from room temperature to $800{ }^{\circ} \mathrm{C}$, under the flow of $20 \mathrm{~mL} \mathrm{~min}^{-1}$ of dried synthetic air $\left(80 \% \mathrm{~N}_{2}\right.$ and $\left.20 \% \mathrm{O}_{2}\right)$. 


\subsubsection{SEM}

SEM analysis was performed on a HITACHI S2600N electron microscope, at $20 \mathrm{keV}$, in secondary electrons fascicle, on samples covered with a thin silver layer. After $24 \mathrm{~h}, 48 \mathrm{~h}$ and $72 \mathrm{~h}$ incubation period, WDs were washed gently with sterile PBS for not disturbing the biofilm, and fixed by immersing each sample in methanol for $5 \mathrm{~s}$. After fixation, samples were allowed to air dry and examined by SEM. Each experiment was performed in triplicate and repeated on at least three separate occasions. For each sample at least three microscopic fields were randomly analyzed by two independent observers. Micrographs considered significant for both observers were selected.

\subsection{Strains and Culture Conditions}

C. albicans ATCC 10231 was purchased from ATCC (American Type Culture Collection, Manassas, VA, USA) and cultured using Sabouraud Agar and Sabouraud broth (Acumedia, Bucharest, Romania). Fungal inoculum was grown overnight in Sabouraud broth and diluted $\sim 1000$ times in the same medium, for reaching a density of $10^{2}-10^{3} \mathrm{CFU} / \mathrm{mL}$.

\subsection{In Vitro Fungal Biofilm Development}

Biofilm formation was assessed in 6 multi-well plates (Nunc, St. Louis, MO, USA), using a static model for monospecific biofilms development. Control WD and MNP@18-SH coated WD pieces of $1 \mathrm{~cm} \times 1 \mathrm{~cm}$ were sterilized by exposure to direct UV light for $20 \mathrm{~min}$ and distributed in 6 multi-well plates (one per well). The C. albicans inoculums $(2 \mathrm{~mL})$ with standardized density were added in each well, to completely cover the WD pieces. Samples were incubated for $24 \mathrm{~h}$ at $37{ }^{\circ} \mathrm{C}$. Biofilms were analyzed by viable cell count assay. Briefly, after $24 \mathrm{~h}$ incubation the culture medium was removed and the pieces of WD washed with sterile PBS (phosphate buffered saline), in order to remove unattached bacteria. WD samples were placed in fresh medium and incubated for other additional $24 \mathrm{~h}$, $48 \mathrm{~h}$ and $72 \mathrm{~h}$. After the incubation period wound dressing pieces were gently washed with sterile PBS for not disturbing the biofilm and placed in $1.5 \mathrm{~mL}$ Eppendorf tubes containing $750 \mu \mathrm{L}$ PBS. Samples were vigorously mixed by vortexing for $30 \mathrm{~s}$ and sonicated for $10 \mathrm{~s}$ in order to disperse biofilm cells into the suspension. Serial ten-fold dilutions were achieved and plated on Sabouraud Agar for viable cell counts assay. Experiments were performed in triplicate and repeated on three separate occasions.

\subsection{Statistical Analysis}

Data were analyzed using GraphPadIn Stat and Prism softwares, by applying One-way Analysis of Variance (ANOVA) test. $p$ values lower than 0.05 were considered significant.

\section{Conclusions}

Classical wound dressings were successfully modified by coating with a novel nanobiosystem based on functionalized magnetite nanoparticles and $\mathrm{SH}$ essential oil. The essential oil was extracted by microwave assisted Neo-Clevenger apparatus and characterized by GC-MS. TEM, XRD, TGA and FT-IR characterization of the fabricated nanostructured coating demonstrated its nanosized 
uniform structure. The biological assay revealed that the newly fabricated nanobiocoating exhibited antimicrobial properties, rendering the wound dressings fibers more resistant to fungal cells adherence and biofilm development. Our results proved that the obtained nanobiocoating combining the excellent properties of iron oxide nanoparticles and the essential oil with antimicrobial properties could represent a novel and successful alternative for inhibiting fungal adhesion and biofilm formation on medical devices and other clinically relevant materials and any surfaces.

\section{Acknowledgments}

This paper is supported by the Sectorial Operational Programme for Human Resources Development, financed by the European Funding Program, under project number POSDRU 107/1.5/S/80765.

\section{Conflicts of Interest}

The authors declare no conflict of interest.

\section{References}

1. Anghel, I.; Holban, A.M.; Grumezescu, A.M.; Andronescu, E.; Ficai, A.; Anghel, A.G.; Maganu, M.; Lazar, V.; Chifiriuc, M.C. Modified wound dressing with phyto-nanostructured coating to prevent staphylococcal and pseudomonal biofilms development. Nanoscale Res. Lett. 2012, 7, 690 .

2. Capoor, M.R.; Sarabahi, S.; Tiwari, V.K.; Narayanan, R.P. Fungal infections in burns: Diagnosis and management. Indian J. Plast. Surg. 2010, 43, S37-S42.

3. Okesola, A.O.; Kehinde, A.O. Bacteriology of non-surgical wound infections in Ibadan, Nigeria. Afr. J. Med. Med. Sci. 2008, 37, 261-264.

4. Toniolo, A.; Endimiani, A.; Luzzaro, F. Microbiology of postoperative infections. Surg. Infect. (Larchmt.) 2006, 7, S13-S16.

5. Anghel, I.; Grumezescu, V.; Andronescu, E.; Anghel, G.A.; Grumezescu, A.M.; Mihaiescu, D.E.; Chifiriuc, M.C. Protective effect of magnetite nanoparticle/Salvia officinalis essential oil hybrid nanobiosystem against fungal colonization on the Provox ${ }^{\circledR}$ voice section prosthesis. Dig. J. Nanomater. Biostruct. 2012, 7, 1205-1212.

6. Saviuc, C.; Holban, A.M.; Grumezescu, A.M.; Bleotu, C.; Banu, O.; Lazar, V.; Mihaiescu, D.E.; Chifiriuc, M.C. Testing antifungal activity of some essential oils using flow cytometry. Lett. Appl. NanoBioSci. 2012, 1, 67-71.

7. Nett, J.; Lincoln, L.; Marchillo, K.; Massey, R.; Holoyda, K.; Hoff, B.; VanHandel, M.; Andes, D. Putative role of $\beta-1,3$ glucans in Candida albicans biofilm resistance. Antimicrob. Agents Chemother. 2007, 51, 510-520.

8. Holban, A.M.; Saviuc, C.; Grumezescu, A.M.; Chifiriuc, M.C.; Banu, O.; Lazar, V. Phenotypic investigation of virulence profiles in some Candida spp. Strains isolated from different clinical specimens. Lett. Appl. Nanobiosci. 2012, 1, 72-76. 
9. Masoudi, A.; Hosseini, H.R.M.; Reyhani, S.M.S.; Shokrgozar, M.A.; Oghabian, M.A.; Ahmadi, R. Long-term investigation on the phase stability, magnetic behavior, toxicity, and MRI characteristics of superparamagnetic Fe/Fe-oxide core/shell nanoparticles. Int. J. Pharm. 2012, 439, 28-40.

10. Azhar, S.L.; Lotfipour, F. Magnetic nanoparticles for antimicrobial drug delivery. Pharmazie 2012, 67, 817-821.

11. Jansch, M.P.; Graf, S.C.; Rühl, E.; Müller, R.H. Adsorption kinetics of plasma proteins on ultrasmallsuperparamagnetic iron oxide (USPIO) nanoparticles. Int. J. Pharm. 2012, 428, 125-133.

12. Huang, C.; Tang, Z.; Zhou, Y.; Zhou, X.; Jin, Y.; Li, D.; Yang, Y.; Zhou, S. Magnetic micelles as a potential platform for dual targeted drug delivery in cancer therapy. Int. J. Pharm. 2012, 429, $113-122$.

13. Grumezescu, A.M.; Andronescu, E.; Ficai, A.; Ficai, D.; Huang, K.S.; Gheorghe, I.; Chifiriuc, M.C. Water soluble magnetic biocomposite with pontetial applications for the antimicrobial therapy. Biointerface Res. Appl. Chem. 2012, 2, 469-475.

14. Andronescu, E.; Grumezescu, A.M.; Ficai, A.; Gheorghe, I.; Chifiriuc, M.C.; Mihaiescu, D.E.; Lazar, V. In vitro efficacy of antibiotic magnetic dextran microspheres complexes against Staphylococcus aureus and Pseudomonas aeruginosa strains. Biointerface Res. Appl. Chem. 2012, 2, 332-338.

15. Anghel, I.; Grumezescu, A.M.; Andronescu, E.; Anghel, A.G.; Ficai, A.; Saviuc, C.; Grumezescu, V.; Vasile, B.S.; Chifiriuc, M.C. Magnetite nanoparticles for functionalized textile dressing to prevent fungal biofilms development. Nanoscale Res. Lett. 2012, 7, 501.

16. Masoudi, A.; Hosseini, H.R.M.; Shokrgozar, M.A.; Ahmadi, R.; Oghabian, M.A. The effect of poly(ethylene glycol) coating on colloidal stability of superparamagnetic iron oxide nanoparticles as potential MRI contrast agent. Int. J. Pharm. 2012, 433, 129-141.

17. Alphandéry, E.; Guyot, F.; Chebbi, I. Preparation of chains of magnetosomes, isolated from Magnetospirillummagneticum strain AMB-1 magnetotactic bacteria, yielding efficient treatment of tumors using magnetic hyperthermia. Int. J. Pharm. 2012, 434, 444-452.

18. Al-Kattan, A.; Girod-Fullana, S.; Charvillat, C.; Ternet-Fontebasso, H.; Dufour, P.; Dexpert-Ghys, J.; Santran, V.; Bordère, J.; Pipy, B.; Bernad, J.; et al. Biomimetic nanocrystallineapatites: Emerging perspectives in cancer diagnosis and treatment. Int. J. Pharm. 2012, 423, 26-36.

19. Grumezescu, A.M.; Andronescu, E.; Ficai, A.; Yang, C.H.; Huang, K.S.; Vasile, B.S.; Voicu, G.; Mihaiescu, D.E.; Bleotu, C. Magnetic nanofluid with antitumoral properties. Lett. Appl. NanoBioSci. 2012, 1, 56-60.

20. Grumezescu, A.M.; Chifiriuc, M.C.; Saviuc, C.; Grumezescu, V.; Hristu, R.; Mihaiescu, D.; Stanciu, G.A.; Andronescu, E. Hybrid nanomaterial for stabilizing the antibiofilm activity of Eugenia carryophyllata essential oil. IEEE Trans. Nanobiosci. 2012, 11, 360-365.

21. Saviuc, C.; Grumezescu, A.M.; Chifiriuc, M.C.; Bleotu, C.; Stanciu, G.; Hristu, R.; Mihaiescu, D.; Lazăr, V. In vitro methods for the study of microbial biofilms. Biointerface Res. Appl. Chem. 2011, 1, 31-40. 
22. Mihaiescu, D.E.; Horja, M.; Gheorghe, I.; Ficai, A.; Grumezescu, A.M.; Bleotu, C.; Chifiriuc, M.C. Water soluble magnetite nanoparticles for antimicrobial drugs delivery. Lett. Appl. NanoBioSci. 2012, 1, 45-49.

23. Zhang, L.; Pornpattananangku, D.; Hu, C.M.; Huang, C.M. Development of nanoparticles for antimicrobial drug delivery. Curr. Med. Chem. 2010, 17, 585-594.

24. Burt, S. Essential oils: Their antibacterial properties and potential applications in foods-A review. Int. J. Food Microbiol. 2004, 94, 223-253.

25. Kavanaugh, N.L.; Ribbeck, K. Selected antimicrobial essential oils eradicate Pseudomonas spp. and Staphylococcus aureus biofilms. Appl. Environ. Microbiol. 2012, 78, 4057-4061.

26. Sefidkon, F.; Abbasi, K.; Khaniki, G.B. Influence of drying and extraction methods on yield and chemical composition of the essential oil of Satureja hortensis. Food Chem. 2006, 99, 19-23.

27. Khajeh, M. Optimization of process variables for essential oil components from Satureja hortensis by supercritical fluid extraction using Box-Behnken experimental design. J. Supercrit. Fluids 2011, 55, 944-948.

28. Skocibusic, M.; Bezic, N.; Dunkic, V. Phytochemical composition and antimicrobial activities of the essential oils from Satureja subspicata V. is. growing in Croatia. Food Chem. 2006, 96, 20-28.

29. Isman, M.B. Plant essential oils for pest and disease management. Crop Protoc. 2000, 19, 603-608.

30. Chifiriuc, C.; Grumezescu, V.; Grumezescu, A.M.; Saviuc, C.; Lazăr, V.; Andronescu, E. Hybrid magnetite nanoparticles/Rosmarinus officinalis essential oil nanobiosystem with antibiofilm activity. Nanoscale Res. Lett. 2012, 7, 209.

31. Grumezescu, A.M.; Saviuc, C.; Chifiriuc, M.C.; Hristu, R.; Mihaiescu, D.E.; Balaure, P.; Stanciu, G.; Lazar, V. Inhibitory activity of $\mathrm{Fe}_{3} \mathrm{O}_{4} /$ oleic acid/usnic acid-core/shell/extra-shell nanofluid on S. aureus biofilm development. IEEE Trans. Nanobiosci. 2011, 10, 269-274.

32. Hadian, J.; Ebrahimi, S.N.; Salehi, P. Variability of morphological and phytochemical characteristics among Satureja hortensis L. accessions of Iran. Ind. Crops Prod. 2010, 32, 62-69.

33. Tozlu, E.; Cakir, A.; Kordali, S.; Tozlu, G.; Ozer, H.; Akcin, T.A. Chemical compositions and insecticidal effects of essential oils isolated from Achillea gypsicola, Satureja hortensis, Origanum acutidens and Hypericum scabrum against broad bean weevil (Bruchus dentipes). Sci. Hortic. 2011, 130, 9-17.

34. Giri, S.K.; Das, N.N.; Pradhan, G.C. Synthesis and characterization of magnetite nanoparticles using waste iron ore tailings for adsorptive removal of dyes from aqueous solution. Colloids Surf. A 2011, 389, 43-49.

35. Maity, D.; Agrawal, D.C. Synthesis of iron oxide nanoparticles under oxidizing environment and their stabilization in aqueous and non-aqueous media. J. Magn. Magn. Mater. 2007, 308, 46-55.

36. Klein, G.; Rüben, C.; Upmann, M. Antimicrobial activity of essential oil components against potential food spoilage microorganisms. Curr. Microbiol. 2013, 67, 200-208.

37. Sahin, F.; Karaman, I.; Güllüce, M.; Oğütçü, H.; Sengül, M.; Adigüzel, A.; Oztürk, S.; Kotan, R. Evaluation of antimicrobial activities of Satureja hortensis L. J. Ethnopharmacol. 2003, 87, 61-65.

38. Saharkhiz, M.J.; Zomorodian, K.; Rezaei, M.R.; Saadat, F.; Rahimi, M.J. Influence of growth phase on the essential oil composition and antimicrobial activities of Satureja hortensis. Nat. Prod. Commun. 2011, 6, 1173-1178. 
39. Mahboubi, M.; Kazempour, N. Chemical composition and antimicrobial activity of Satureja hortensis and Trachyspermum copticum essential oil. Iran. J. Microbiol. 2011, 3, 194-200.

40. Sabzghabaee, A.M.; Davoodi, N.; Ebadian, B.; Aslani, A.; Ghannadi, A. Clinical evaluation of the essential oil of "Satureja Hortensis" for the treatment of denture stomatitis. Dent. Res. J. (Isfahan) 2012, 9, 198-202.

41. Marcos-Arias, C.; Eraso, E.; Madariaga, L.; Quindós, G. In vitro activities of natural products against oral Candida isolates from denture wearers. BMC Complement. Altern. Med. 2011, 11, 119.

42. Boyraz, N.; Ozcan, M. Inhibition of phytopathogenic fungi by essential oil, hydrosol, ground material and extract of summer savory (Satureja hortensis L.) growing wild in Turkey. Int. J. Food Microbiol. 2006, 107, 238-242.

43. Delgado, B.; Fernandez, P.S.; Palop, A.; Periago, P.M. Effect of thymol and cymene on Bacillus cereus vegetative cells evaluated through the use of frequency distributions. Food Microbiol. 2004, 21, 327-334.

44. Lambert, R.J.W.; Skandamis, P.N.; Coote, P.J.; Nychas, G.J.E. A study of the minimum inhibitory concentration and mode of action of oregano essential oil, thymol and carvacrol. J. Appl. Microbiol. 2001, 91, 453-462.

45. Saviuc, C.; Grumezescu, A.M.; Bleotu, C.; Holban, A.; Chifiriuc, C.; Balaure, P.; Lazar, V. Phenotipical studies for raw and nanosystem embedded Eugenia carryophyllata buds essential oil effect on Pseudomonas aeruginosa and Staphylococcus aureus strains. Biointerface Res. Appl. Chem. 2011, 1, 111-118.

46. Grumezescu, A.M.; Holban, A.M.; Andronescu, E.; Tomoiaga, M.; Ficai, A.; Bleotu, C.; Chifiriuc, M.C. Microbiological applications of a new water dispersible magnetic nanobiocomposite. Lett. Appl. NanoBioSci. 2012, 1, 83-90.

47. Grumezescu, A.M.; Andronescu, E.; Voicu, G.; Huang, K.S.; Ficai, A.; Yang, C.H.; Bleotu, C.; Chifiriuc, M.C. Antitumor activity of magnetite nanoparticles: Influence of hydrocarbonated chain of saturated aliphatic monocarboxylic acids. Curr. Org. Chem. 2013, 17, 831-840.

(C) 2013 by the authors; licensee MDPI, Basel, Switzerland. This article is an open access article distributed under the terms and conditions of the Creative Commons Attribution license (http://creativecommons.org/licenses/by/3.0/). 